\title{
Representing Local Structure Using Tensors II
}

\author{
Hans Knutsson ${ }^{1,2}$, Carl-Fredrik Westin ${ }^{1,3}$, and Mats Andersson ${ }^{1,2}$ \\ 1 Department of Biomedical Engineering, Linköping University, Sweden \\ 2 Center for Medical Image Science and Visualization (CMIV), Linköping, Sweden \\ 3 Laboratory of Mathematics in Imaging, Brigham and Women's Hospital, Harvard \\ Medical School, Boston, MA \\ knutte@imt.liu.se
}

\begin{abstract}
Estimation of local spatial structure has a long history and numerous analysis tools have been developed. A concept that is widely recognized as fundamental in the analysis is the structure tensor. However, precisely what it is taken to mean varies within the research community. We present a new method for structure tensor estimation which is a generalization of many of it's predecessors. The method uses filter sets having Fourier directional responses being monomials of the normalized frequency vector, one odd order sub-set and one even order sub-set. It is shown that such filter sets allow for a particularly simple way of attaining phase invariant, positive semi-definite, local structure tensor estimates. We continue to compare a number of known structure tensor algorithms by formulating them in monomial filter set terms. In conclusion we show how higher order tensors can be estimated using a generalization of the same simple formulation.
\end{abstract}

Keywords: structure tensor, higher order, quadrature, monomial filter.

\section{Introduction}

Many of the popular image analysis concepts of today have roots that can be traced to early work in signal processing and optics, e.g. Riesz transforms, 1], Zernike moments, [2], and Gabor signals, 3]. The first steps towards analysis of digital images were taken more then four decades ago 4]. From the very start detecting edges and lines in images was considered a fundamental operation [5]. Since these early days new and more advanced schemes for analysis of local image structure has been suggested in a seemingly never ending stream. Papers having a particular relevance in the present context can be found in, for example [6] - 33. Local image orientation, scale, frequency, phase, motion and locality of estimates are prominent examples of features that have been considered central in the analysis.

The main force driving the research has been the need for an efficient and useful analysis of data produced by increasingly capable imaging devices. Both the outer and the inner dimensionality is commonly high, e.g. volume sequence data and tensor field data respectively. Regardless of this development the first stages in the analysis remain the same. In most cases the processing starts by

A. Heyden and F. Kahl (Eds.): SCIA 2011, LNCS 6688, pp. 545 556 2011.

(C) Springer-Verlag Berlin Heidelberg 2011 
performing local linear combinations of image values, e.g. convolution operators. The output from these convolutions are then usually combined in a non-linear fashion to produce local feature descriptors.

We will focus on developments regarding a particular instance of such algorithms, local structure tensor estimation. We start by presenting a general estimation scheme using monomial filter sets.

\section{Monomial Filters}

The monomial filters are spherically separable, i.e. defined as a product of one radial and one directional part:

$$
F(\hat{\boldsymbol{\mu}})=R(\rho) D(\hat{\boldsymbol{\mu}})
$$

where $\boldsymbol{\mu}$ defines the Fourier domain (FD) coordinates and $\rho=\|\boldsymbol{\mu}\|$. The radial part, $R(\rho)$, is a band-pass or high-pass filter $(R(0)=0)$ and a typical choice is the lognormal function [9].

Directional part - The directional part consists of monomials i.e. products of positive integer powers of the components of $\hat{\boldsymbol{\mu}}$. Performing $n$ repeated outer products of $\hat{\boldsymbol{\mu}}$ will contain all order $n$ component products.

$$
\hat{\boldsymbol{\mu}}^{\otimes n}=\underbrace{\hat{\boldsymbol{\mu}} \otimes \hat{\boldsymbol{\mu}} \ldots \otimes \hat{\boldsymbol{\mu}}}_{n \text { entities }}
$$

For convenience we rearrange the terms such that the directional part, $\boldsymbol{D}_{n}(\hat{\boldsymbol{\mu}})$, becomes a matrix:

$$
\boldsymbol{D}_{n}(\hat{\boldsymbol{\mu}})=\hat{\boldsymbol{\mu}}\left\lfloor\hat{\boldsymbol{\mu}}^{\otimes(n-1)}\right\rfloor^{T}
$$

Here the " $\lfloor$ 」" notation implies a lineup operation which arrange the elements of a multi dimensional array into a lexicographic ordered column vector. The motivation for introducing this notation is that letting $\boldsymbol{D}_{n}$ contain the elements of $\hat{\boldsymbol{\mu}}^{\otimes n}$ arranged as a matrix greatly simplifies the equations needed in the following analysis.

To handle the special cases of $n=0$ and $n=1$ we introduce the following definitions:

$$
\hat{\boldsymbol{\mu}}\left\lfloor\hat{\boldsymbol{\mu}}^{\otimes(-1)}\right\rfloor \equiv \hat{\boldsymbol{\mu}}^{\otimes 0} \equiv \mathbf{I}
$$

where $\mathbf{I}$ is an identity matrix scaled to have a unity frobenius norm. The need to introduce these definitions correspond to the fact that order 0 implies a scalar entity that does not carry orientation information and thus constitutes a special case.

It is also worth noting here that the odd part of $\boldsymbol{D}_{1}(\hat{\boldsymbol{\mu}})$ corresponds to the Hilbert transform in the 1-dimensional case and the Riesz transform for higher dimensions, [1].

The monomial filter matrix - For each order $n$ a monomial filter matrix is defined as:

$$
\boldsymbol{F}_{n}=R(\rho) \boldsymbol{D}_{n}(\hat{\boldsymbol{\mu}})
$$


FD

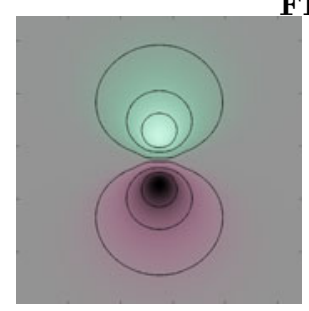

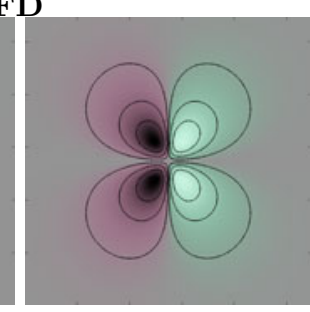

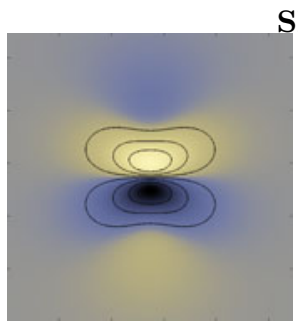

SD

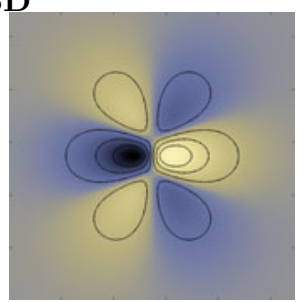

Fig. 1. Left: Fourier domain images of $F_{3}(1,1)$ and $F_{3}(1,2)$, see eq. (6). High values are bright and low values are dark. Green indicates positive real values and red indicates negative real values. Right: Spatial domain images of $F_{3}(1,1)$ and $F_{3}(1,2)$. Yellow indicates positive imaginary values and blue indicates negative imaginary values. The black contours are iso-level lines. The remaining filters in the set are $90 \mathrm{deg}$ rotated copies of the shown filters.

As an example consider the monomial filter matrix of order three in 2D. Using the notation $\hat{\boldsymbol{\mu}}=(\mu, \nu)^{T}$ the monomial filter matrix is computed as:

$$
\begin{aligned}
\boldsymbol{F}_{3} & \left.=R(\rho)\left(\begin{array}{l}
\mu \\
\nu
\end{array}\right) \mid \begin{array}{ll}
\mu^{2} & \mu \nu \\
\mu \nu & \nu^{2}
\end{array}\right]^{T} \\
& =R(\rho)\left(\begin{array}{c}
\mu \\
\nu
\end{array}\right)\left(\begin{array}{lll}
\mu^{2} & \mu \nu & \mu \nu \nu^{2}
\end{array}\right) \\
& =R(\rho)\left(\begin{array}{cccc}
\mu^{3} & \mu^{2} \nu & \mu^{2} \nu & \mu \nu^{2} \\
\mu^{2} \nu & \mu \nu^{2} & \mu \nu^{2} & \nu^{3}
\end{array}\right)
\end{aligned}
$$

For clarity of the presentation most examples in this paper are given in $2 \mathrm{D}$. The proposed concept is, however, valid for any signal dimensions.

Monomial filter responses - Now let the spatial domain (SD) correspondence of the monomial filter matrix $\boldsymbol{F}_{n}$ be denoted $\mathbf{F}_{n}$. Each element of $\mathbf{F}_{n}$ consequently contains the convolution kernel of the corresponding FD filter function in $\boldsymbol{F}_{n}$. If the multi dimensional signal is denoted $\mathbf{s}(\mathbf{x})$ where $\mathbf{x}$ denotes the SD coordinates the monomial filter response matrix, $\mathbf{Q}_{n}(\mathbf{x})$, is defined as:

$$
\mathbf{Q}_{n}(\mathbf{x})=\mathbf{F}_{n}(\mathbf{x}) * \mathrm{~s}(\mathbf{x})
$$

Denoting the Fourier transform of $\mathbf{s}$ around $\mathbf{x}$ by $\mathrm{S}_{\mathbf{x}}$ the same relation is, in the Fourier domain, expressed as:

$$
\mathbf{Q}_{n}(\mathbf{x})=\int \boldsymbol{F}_{n}(\boldsymbol{\mu}) \mathrm{S}_{\mathbf{x}}(\boldsymbol{\mu}) d \boldsymbol{\mu}
$$

In this general description each element of $\mathbf{Q}_{n}(\mathbf{x})$ contains the monomial filter responses for the entire signal. Since all filtering operations in this paper are shift invariant we may from now on sometimes omit the spatial coordinate vector $\mathbf{x}$ 
and, when doing so, consider each element of $\mathbf{Q}_{n}$ to contain a monomial filter responses for any given spatial coordinate.

\section{Signal Classes}

It is useful in the following to define different classes of signals. We will here define three different signal classes: Sinusoidal, Simple and Rank $d$ signals.

Sinusoidal signals - We first present the simplest case, a sinusoidal signal with amplitude a, spatial frequency $\boldsymbol{u}$, and phase $\theta$. For this case the monomial filter response matrix can be described in FD terms as:

$$
\mathrm{s}(\mathbf{x})=\mathrm{a} \cos \left(\boldsymbol{u}^{T} \mathbf{x}+\theta\right)
$$

both even and odd order filters will respond and we get:

$$
\mathbf{Q}_{n}=\left\{\begin{aligned}
a \cos (\theta) R(\rho) \boldsymbol{D}_{n}(\hat{\boldsymbol{u}}) & \text { for even } n \\
-i \operatorname{a} \sin (\theta) R(\rho) \boldsymbol{D}_{n}(\hat{\boldsymbol{u}}) & \text { for odd } n
\end{aligned}\right.
$$

Simple signals - Following [18] we define signals that can be expressed by equation (11) to be termed simple signals.

$$
\mathrm{s}(\mathbf{x})=\mathrm{g}\left(\hat{\boldsymbol{u}}^{T} \mathbf{x}\right)
$$

Here $\mathrm{g}(\mathrm{x})$ is any real, one variable, function and $\mathrm{x}=\hat{\boldsymbol{u}}^{T} \mathbf{x} . \quad \hat{\boldsymbol{u}}$ is a unit vector giving the orientation of the signal. For this case the monomial filter response matrix can be described in FD terms as:

$$
\begin{aligned}
\mathbf{Q}_{n}(\hat{\boldsymbol{u}}) & =\mathrm{A}_{n} \boldsymbol{D}_{n}(\hat{\boldsymbol{u}}) \\
& =\mathrm{A}_{n} \hat{\boldsymbol{u}}\left\lfloor\hat{\boldsymbol{u}}^{\otimes(n-1)}\right\rfloor^{T}
\end{aligned}
$$

The local orientation invariant filter factor, $\mathrm{A}_{n}$, is a function of the radial filter function, $R(\rho)$, and the signal generating function, $\mathrm{g}(\mathrm{x})$. The fact that the Fourier transform of a simple signal is non-zero only on a line through the origin makes for a simple solution. Denoting the Fourier transform of $\mathrm{g}(\mathrm{x})$ by $\mathrm{G}(u)$ we find the filter response amplitude as:

$$
\begin{cases}\mathrm{A}_{n}=\mathrm{A}_{\mathrm{e}}=\int R(|u|) \mathrm{G}(u) d u & \text { for even } n \\ \mathrm{~A}_{n}=\mathrm{A}_{\circ}=\int R(|u|) \mathrm{G}(u) \operatorname{sign}(u) d u & \text { for odd } n\end{cases}
$$

Unless explicitly mentioned all signals will in the following be regarded simple.

Rank $d$ signals - Although not directly used here, it is worth noting that it is straight forward to classify more complex signals in a similar manner. Let $\hat{\boldsymbol{U}}$ be a projection operator of rank $d$ and $\mathrm{g}$ be a real function of $d$ variables, then

$$
\mathrm{s}(\mathbf{x})=\mathrm{g}(\hat{\boldsymbol{U}} \mathbf{x})
$$

is a rank $d$ signal. Thus, a simple signal is a rank one signal. A full rank signal corresponds to $\hat{\boldsymbol{U}}=\hat{\boldsymbol{I}}$. In the following we will only distinguish between sinusoidal, simple and non-simple signals. 


\section{Second Order Structure Tensors}

The next step towards obtaining a structure tensor is to compute the outer product of the filter matrix.

$$
\mathbf{T}_{n}^{2}=\mathbf{Q}_{n} \mathbf{Q}_{n}^{T}
$$

where " $T$ " denotes complex conjugate transpose.

We will present the case where the local neighborhood of the image consists of a simple signal with direction $\hat{\boldsymbol{u}}$. As a simple introductory example we consider 2-dimensional monomial filters of order three.

$$
\mathbf{Q}_{3}=\mathrm{A}_{\circ}\left(\begin{array}{cccc}
u^{3} & u^{2} v & u^{2} v & u v^{2} \\
u^{2} v & u v^{2} & u v^{2} & v^{3}
\end{array}\right)
$$

Carrying out the sums we get:

$$
\begin{aligned}
\mathbf{T}_{3} & =\mathbf{Q}_{3} \mathbf{Q}_{3}^{T} \\
& =\mathrm{A}_{\circ} \mathbf{A}_{\circ}^{*}\left(\begin{array}{cccc}
u^{3} & u^{2} v & u^{2} v & u v^{2} \\
u^{2} v & u v^{2} & u v^{2} & v^{3}
\end{array}\right)\left(\begin{array}{cc}
u^{3} & u^{2} v \\
u^{2} v & u v^{2} \\
u^{2} v & u v^{2} \\
u v^{2} & v^{3}
\end{array}\right) \\
& =\left|\mathrm{A}_{\circ}\right|^{2} \underbrace{\left(u^{2}+v^{2}\right)^{2}}_{=1}\left(\begin{array}{cc}
u^{2} & u v \\
u v & v^{2}
\end{array}\right) \\
& =\left|\mathrm{A}_{\circ}\right|^{2}\left(\begin{array}{cc}
u^{2} & u v \\
u v & v^{2}
\end{array}\right)
\end{aligned}
$$

In general the matrix product of equation (15) becomes:

$$
\begin{aligned}
\mathbf{T}_{n}^{2} & =\underbrace{\mathrm{A}_{n} \hat{\boldsymbol{u}}\left\lfloor\hat{\boldsymbol{u}}^{\otimes(n-1)}\right\rfloor^{T}}_{\mathbf{Q}_{n}} \underbrace{\left\lfloor\hat{\boldsymbol{u}}^{\otimes(n-1)}\right\rfloor \hat{\boldsymbol{u}}^{T} \mathrm{~A}_{n}^{*}}_{\mathbf{Q}_{n}^{T}} \\
& =\mathrm{A}_{n} \hat{\boldsymbol{u}} \underbrace{\left\lfloor\hat{\boldsymbol{u}}^{\otimes(n-1)}\right\rfloor^{T}\left\lfloor\hat{\boldsymbol{u}}^{\otimes(n-1)}\right\rfloor}_{\text {inner product }} \hat{\boldsymbol{u}}^{T} \mathrm{~A}_{n}^{*}
\end{aligned}
$$

The inner product of the lined up outer products above can be performed in reversed order. Then, for $n>0$, equation (18) simplifies to:

$$
\mathbf{T}_{n}^{2}=\mathrm{A}_{n} \hat{\boldsymbol{u}} \underbrace{\left(\hat{\boldsymbol{u}}^{T} \hat{\boldsymbol{u}}\right)^{(n-1)}}_{=1} \hat{\boldsymbol{u}}^{T} \mathrm{~A}_{n}^{*}
$$

By definition $\hat{\boldsymbol{u}}^{T} \hat{\boldsymbol{u}}$ is equal to one. It follows that the under-braced term also equals one and we have the desired result:

$$
\mathbf{T}_{n}^{2}=\left|\mathrm{A}_{n}\right|^{2} \hat{\boldsymbol{u}} \hat{\boldsymbol{u}}^{T} ; \quad n>0
$$

For $n=0$ we have a special case since the filter is isotropic, $\boldsymbol{D}_{n}=1$, and $\mathbf{T}_{0}^{2}=\mathbf{Q}_{0} \mathbf{Q}_{0}^{T}=\left|\mathrm{A}_{\mathrm{e}}\right|^{2} \mathbf{I}$. 
Monomial quadrature - As stated in equation (13) even and odd filters will have different local magnitudes.

$$
\mathbf{T}_{n}^{2}=\left\{\begin{array}{cc}
\mathrm{A}_{\mathrm{e}}^{2} \hat{\boldsymbol{u}} \hat{\boldsymbol{u}}^{T} & \text { for even } n \\
\left|\mathrm{~A}_{\circ}\right|^{2} \hat{\boldsymbol{u}} \hat{\boldsymbol{u}}^{T} & \text { for odd } n
\end{array}\right.
$$

A phase invariant monomial quadrature tensor can now be computed as the sum of one even index tensor and odd index tensor. Letting $n$ be even and $m$ odd we obtain:

$$
\begin{aligned}
\mathbf{T}_{n m}^{2} & =\mathbf{T}_{n}^{2}+\mathbf{T}_{m}^{2} \\
& =\mathbf{Q}_{n} \mathbf{Q}_{n}^{T}+\mathbf{Q}_{m} \mathbf{Q}_{m}^{T} \\
& =\left|\mathrm{A}_{\mathrm{e}}+\mathrm{A}_{\circ}\right|^{2} \hat{\boldsymbol{u}} \hat{\boldsymbol{u}}^{T} \\
& =\mathrm{q}^{2} \hat{\boldsymbol{u}} \hat{\boldsymbol{u}}^{T}
\end{aligned}
$$

Note that the tensor magnitude, $\mathbf{q}$, will be the same regardless of the order of the filters used.

Tensor positivity - A more compact expression of the monomial quadrature tensor can be attained by concatenating the even and the odd filter response matrices to a single matrix. The ", " notation implies concatenation of the arguments left to right.

$$
\mathbf{Q}_{n m}=\left(\mathbf{Q}_{n}, \mathbf{Q}_{m}\right)
$$

and compute the monomial quadrature tensor of origin $(m, n)$ as

$$
\mathbf{T}_{n m}^{2}=\mathbf{Q}_{n m} \mathbf{Q}_{n m}^{T}
$$

As the monomial quadrature tensor is computed from products of filter response matrices

$$
\mathbf{T}_{n m}^{2}=\mathbf{Q}_{n m} \mathbf{Q}_{n m}^{T}=\sum_{k} \lambda_{k} \hat{\mathbf{e}}_{k} \hat{\mathbf{e}}_{k}^{T}
$$

it follows that all $\lambda_{k} \geq 0$ which allows for robust certainty estimates for the local structure estimation.

\section{Structure Tensor Estimation Variations}

Local structure analysis algorithms are quite complex and involve a lot more than the filters used. This makes comparisons difficult to interpret from a filter point of view. There are, however, a number of interesting similarities between different suggested algorithms. A few previous comparisons can be found in [21] and 26. In the following we point out the relation to the monomial approach for a number of well known approaches to structure tensor estimation. We show that nearly all variants can be formulated as special or modified versions of the monomial approach. 
The structure tensor, $\mathbf{T}$ - The first publications mentioning tensors as a representation for local orientation and structure is due to Knutsson, [1216. Similar to the earlier developed vector representation, 911, the construction is based on a set of quadrature filters oriented in a number of fixed orientations, $\mathrm{q}_{k}, k$ indicating the orientation. The structure tensor is obtained as:

$$
\mathbf{T}=\sum_{k} \sqrt{\mathbf{q}_{k} \mathbf{q}_{k}^{*}} \mathbf{T}_{k}
$$

The vector variant works for two dimensional signals but for three dimension, or more, the tensor formulation is necessary. The loglet based structure tensor estimation suggested in 22 also uses this weighted 'basis tensor' approach but involve a different set of filters allowing higher order orientations components to be incorporated.

Unlike the methods discussed below these method for structure tensor construction are not possible to describe as a special or modified case of the monomial approach.

The gradient tensor, $\mathbf{T}_{\mathrm{G}}$ - The simplest way to obtain a matrix describing local orientation is exemplified by Bigun-Granlund's inertia matrix [13] and Förstners corner detector [15]. This matrix is constructed as the outer product of the local gradient and is, in the notation introduced above and $\mathbf{Q}$ defined by equation(7), given by:

$$
\begin{aligned}
\mathbf{T}_{\mathbf{G}} & =\mathbf{T}_{1} \\
& =\mathbf{Q}_{1} \mathbf{Q}_{1}^{T}
\end{aligned}
$$

Although the authors never mention tensors in the original work this outer product matrix estimate is often referred to as the gradient tensor or the structure tensor.

Since only a single order, i.e. order one, is used this tensor is not phase invariant. Another drawback is that the frequency bandwidth of the estimate can become twice that of the original signal which may cause significant aliasing artifacts. Both these shortcomings are in practice, to some extent, remedied by the use of an averaging filter performing a weighted summation of local outer products. On the other hand this decreases the spatial resolution of the estimate, 24].

The boundary tensor, $\mathbf{T}_{\mathrm{B}}$ - The boundary tensor originally suggested by Köthe, [23], uses orders one and two and constitutes a special case of the monomial quadrature tensor.

$$
\begin{aligned}
\mathbf{T}_{\mathbf{B}} & =\mathbf{T}_{12} \\
& =\mathbf{Q}_{12} \mathbf{Q}_{12}^{T}
\end{aligned}
$$

The energy tensor, $\mathbf{T}_{\mathrm{E}}$ - The energy tensor, suggested by Felsberg, 28, is a variant where filters of different orders are involved in the computed products. 
The energy tensor uses an isotropically bandpass filter signal, it's gradient and it's Hessian. It can in monomial terms be expressed as:

$$
\begin{aligned}
\mathbf{T}_{\mathbf{E}} & =\mathbf{T}_{1}+\mathbf{T}_{(0,2)} \\
& =\mathbf{Q}_{1} \mathbf{Q}_{1}^{T}+\mathrm{q}_{0} \mathbf{Q}_{2}
\end{aligned}
$$

Note that the filter response matrices here have different radial frequency response and the mixing of different order terms will not give a positive semidefinite tensor for all image neighborhoods.

Gradient energy tensor, $\mathbf{T}_{\mathrm{GE}}$ - The gradient energy tensor (GET) suggested in 29] can be said to use the same formula as the energy tensor with the input signal replaced by it's gradient. In monomial terms the result can be expressed:

$$
\mathbf{T}_{\mathrm{GE}}=\mathbf{Q}_{2} \mathbf{Q}_{2}^{T}+\frac{1}{2}\left(\mathbf{Q}_{1} \mathbf{Q}_{3}^{T}+\mathbf{Q}_{3} \mathbf{Q}_{1}^{T}\right)
$$

Note that also in this case the filter response matrices here have different radial frequency response and that the mixing of different order terms will not give a positive semi-definite tensor for all image neighborhoods.

Spatial 2:nd order polynomial tensor, $\mathbf{T}_{\text {SP }}$ - The 2:nd order polymer tensor suggested by Farnebäck in [19] is a sum of outer products of 1:st and 2:nd order monomial filters. The difference from the monomial approach is that the filters are designed as windowed 1:st and 2:nd order polynomials in the spatial domain.

$$
\begin{aligned}
\mathbf{T}_{\mathbf{S P}} & =\mathbf{T}_{1}+\mathbf{T}_{2} \\
& =\mathbf{Q}_{1} \mathbf{Q}_{1}^{T}+\mathbf{Q}_{2} \mathbf{Q}_{2}^{T}
\end{aligned}
$$

This spatial design results in 1:st and 2:nd order filter that have different radial functions in the frequency domain. For this reason the result is not in general phase invariant i.e components are not in quadrature. However, since it is a sum of squares, the result is always positive semi-definite.

Spherical harmonics, $\mathbf{T}_{\text {SH }}$ - A somewhat different way to estimate a local structure tensor is suggested in 24]. This approach is based on sums of products of spherically separable filters. The filter have the same radial function and the directional functions are spherical harmonic functions. The structure tensor carries information about 0 :th and 2 :nd order variations in orientation. A product between an order $j$ filter and an order $k$ filter will contain signal components of orders $j-k$ and $j+k$. By an appropriate weighted summation of a number of filter products it is possible to retain only order 0 and order 2 in the correct proportion while canceling out all other orders: i.e:

$$
\mathbf{T}_{\mathbf{S H}}=\sum_{j k} w_{j k} \mathbf{H}_{j} \mathbf{H}_{k}
$$

This is a very general approach and, since spherical harmonic filter sets of orders 1 to $N$ span the same function space as monomial filter sets of orders 1 to $N$, 
all monomial tensor variations can also be expressed in this way. With proper weights the result can also be made phase-invariant.

Sum of monomial tensors - Even more careful weighting of spherical harmonic filter products will give positive semi-definite tensors, in this case the result will be equivalent to a sum of tensor estimates over different order, $n \geq 0$ (even and odd), monomial filter matrices, $\mathbf{Q}_{n}$.

$$
\mathbf{T}_{\mathbf{S M}}=\sum_{n} w_{n} \mathbf{Q}_{n} \mathbf{Q}_{n}^{T}
$$

\section{Higher Order Structure Tensors}

In equation (18) the filter matrix is constructed to produce a 2:nd order tensor. However, a simple rearrangement of the order $n$ filter matrix components will allow tensors of order $2 p$ to be estimated.

$$
\begin{aligned}
& \mathbf{T}_{\mathbf{2} \mathbf{p}, n}^{2}=\underbrace{\mathrm{A}_{n}\left\lfloor\hat{\boldsymbol{u}}^{\otimes p}\right\rfloor\left\lfloor\hat{\boldsymbol{u}}^{\otimes(n-p)}\right\rfloor^{T}}_{\mathbf{Q}_{(p) n}} \underbrace{\left\lfloor\hat{\boldsymbol{u}}^{\otimes(n-p)}\right\rfloor\left\lfloor\hat{\boldsymbol{u}}^{\otimes p}\right\rfloor^{T} \mathrm{~A}_{n}}_{\mathbf{Q}_{(p) n}^{T}} \\
& =\mathrm{A}_{n}\left\lfloor\hat{\boldsymbol{u}}^{\otimes p}\right\rfloor \underbrace{\left\lfloor\hat{\boldsymbol{u}}^{\otimes(n-p)}\right\rfloor^{T}\left\lfloor\hat{\boldsymbol{u}}^{\otimes(n-p)}\right\rfloor}_{\text {inner product }}\left\lfloor\hat{\boldsymbol{u}}^{\otimes p}\right\rfloor^{T} \mathrm{~A}_{n}
\end{aligned}
$$

As before the inner product of the lined up outer products above can be performed in reversed order. Then, for $n \geq p \geq 0$, equation (18) simplifies to:

$$
\mathbf{T}_{2 \mathbf{p}, n}^{2}=\mathrm{A}_{n}\left\lfloor\hat{\boldsymbol{u}}^{\otimes p}\right\rfloor \underbrace{\left(\hat{\boldsymbol{u}}^{T} \hat{\boldsymbol{u}}\right)^{(n-p)}}_{=1}\left\lfloor\hat{\boldsymbol{u}}^{\otimes p}\right\rfloor^{T} \mathrm{~A}_{n}
$$

By definition $\hat{\boldsymbol{u}}^{T} \hat{\boldsymbol{u}}$ is equal to one. It follows that the under-braced term also equals one which gives:

$$
\mathbf{T}_{\mathbf{2 p}, n}^{2}=\left|\mathbf{A}_{n}\right|^{2}\left\lfloor\hat{\boldsymbol{u}}^{\otimes p}\right\rfloor\left\lfloor\hat{\boldsymbol{u}}^{\otimes p}\right\rfloor^{T} ; \quad n \geq p \geq 0
$$

The result now holds the components of a tensor of order $2 p$. However, due to the use of the lineup operator, the components are stored in matrix form and they need to be re-organized in order to obtain the result as a proper tensor $\boldsymbol{\Upsilon}$ of order $2 p$.

$$
\boldsymbol{\Upsilon}_{2 \mathbf{p}, n}=\left\lceil\mathbf{T}_{2 \mathbf{p}, n}^{2}\right\rceil
$$

The " $\rceil$ " notation used here indicates a reshape operation that restores the proper structure of the data, i.e. the result is a tensor having $2 p$ indexes.

As 2:nd order tensors are naturally represented as matrices the lineup operator greatly simplifies the notation. For higher order tensors, however, the use of standard tensor notation may be preferred by some readers. Equations (36) and (37) can then be jointly expressed as:

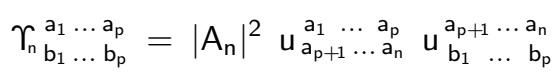


According to the Einstein convention a summation is performed over equal indexes and equation 38 clearly shows that the difference between tensors of different order is how many indexes are summed over (contracted). Letting $p=0$ means summing over all indexes and the result is a scalar representing the local energy. For $p=1$ we obtain the standard structure tensor. For $p>1$ we obtain higher order tensors having the power to represent more complex local structure.

Non-simple signals - For such non-simple signals equation (38) is no longer applicable since there is no unique local orientation, $\boldsymbol{u}$. Directly expressed as a sum of filter products, corresponding to a generalization of the monomial filter response matrix product in equation (15), we obtain an order $2 p$ structure tensor as:

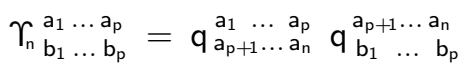

Tensors of order four have been used to analyze situations with two orientations present, e.g. 25/3133. Applications where tensors of order higher than four have been used are so far not known to the authors but can be expected to prove a powerful tool when more that two orientation are present.

To produce quadrature type tensors we still need to add a tensor from odd order filter sets and a tensor from even order filter sets. In the most general case we can express the estimation of local structure tensors of order $2 p$ as a weighted summation of order $2 p$ tensors, here of order $\left(\begin{array}{l}p \\ p\end{array}\right)$, from monomial filter sets of different orders, i.e:

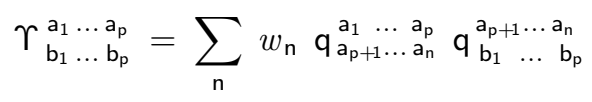

\section{Conclusion}

Research concerning 2:nd order structure tensor estimation is still continuing after more than two decades. The higher order tensor estimates produced by equation (40) contains a much richer representation of the local structure and we expect that the future will hold considerable effort towards fully understanding these new higher order constructs.

\section{Acknowledgment}

The Swedish Research Council and Linköpings Universitet are gratefully acknowledged for supporting this work.

\section{References}

1. Riesz, M.: Sur les fonctions conjuge'es. Math. Zeit. 27, 218-244 (1927)

2. Zernike, F.: Diffraction theory of the cut procedure and its improved form, the phase contrast method. Physica 1, 689-704 (1934) 
3. Gabor, D.: Theory of communication. J. Inst. Elec. Eng. 93(26), 429-457 (1946)

4. Hu, M.K.: Visual pattern recognition by moment invariants. IRE Transactions on Information Theory, IT-8(2), 179-187 (1962)

5. Roberts, L.G.: Machine Perception of three-dimensional Solid. In: Tippell, J.T. (ed.) Optical and Electro-Optical Information Processing, pp. 159-197. MIT Press, Cambridge (1965)

6. Granlund, G.H.: In search of a general picture processing operator. Computer Graphics and Image Processing 8(2), 155-178 (1978)

7. Danielsson, P.E.: Rotation invariant operators with directional response. In: Proceedings 5'th Int. Conf. on Pattern Recognition, Miami Beach, Florida (1980)

8. Knutsson, H., Wilson, R.G., Granlund, G.H.: Anisotropic filtering operations for image enhancement and their relation to the visual system. In: IEEE Computer Society Conference on Pattern Recognition and Image Processing, Dallas, Texas (August 1981)

9. Knutsson, H.: Filtering and Reconstruction in Image Processing. PhD thesis, Linköping University, Sweden, Diss. No. 88 (1982)

10. Knutsson, H., Granlund, G.H.: Texture analysis using two-dimensional quadrature filters. In: IEEE Computer Society Workshop on Computer Architecture for Pattern Analysis and Image Database Management - CAPAIDM, Pasadena (October 1983)

11. Knutsson, H.: Producing a continuous and distance preserving 5-D vector representation of 3-D orientation. In: IEEE Computer Society Workshop on Computer Architecture for Pattern Analysis and Image Database Management - CAPAIDM, pp. 175-182, Miami Beach, Florida, November 1985. IEEE. Report LiTH-ISY-I0843, Linköping University, Sweden (1986)

12. Knutsson, H.: A tensor representation of 3-D structures. In: 5th IEEE-ASSP and EURASIP Workshop on Multidimensional Signal Processing, Noordwijkerhout, The Netherlands (September 1987), poster presentation

13. Bigün, J., Granlund, G.H.: Optimal orientation detection of linear symmetry. In: IEEE First International Conference on Computer Vision, London, Great Britain, pp. 433-438 (June 1987)

14. Lenz, R.: Rotation-invariant operators and scale space filtering. Pattern Recognition Letters 6, 151-154 (1987)

15. Forstner, W., Gulch, E.: A fast operator for detection and precise location of distinct points, corners and centres of circular features. In: ISPRS Intercommission Conference on Fast Processing of Photogrammetric Data, pp. 281-305 (1987)

16. Knutsson, H.: Representing local structure using tensors. In: The 6th Scandinavian Conference on Image Analysis, Oulu, Finland, pp. 244-251, (June 1989); Report LiTH-ISY-I-1019, Computer Vision Laboratory, Linköping University, Sweden

17. Knutsson, H., Bårman, H., Haglund, L.: Robust orientation estimation in 2D, 3D and 4D using tensors. In: Proceedings of Second International Conference on Automation, Robotics and Computer Vision, ICARCV 1992, Singapore (September 1992)

18. Granlund, G.H., Knutsson, H.: Signal Processing for Computer Vision. Kluwer Academic Publishers, Dordrecht (1995) ISBN 0-7923-9530-1

19. Farnebäck, G.: Fast and accurate motion estimation using orientation tensors and parametric motion models. In: Proceedings of 15th International Conference on Pattern Recognition, vol. 1, pp. 135-139. IAPR, Barcelona (2000)

20. Felsberg, M., Sommer, G.: The monogenic signal. IEEE Transactions on Signal Processing 49(12), 3136-3144 (2001) 
21. Johansson, B., Farnebäck, G.: A theoretical comparison of different orientation tensors. In: Proceedings SSAB 2002 Symposium on Image Analysis, pp. 69-73. SSAB, Lund (2002)

22. Knutsson, H., Andersson, M.: Loglets: Generalized quadrature and phase for local spatio-temporal structure estimation. In: Proceedings of the Scandinavian Conference on Image Analysis (SCIA) (June 2003)

23. Köthe, U.: Inegrated edge and junction detection with the boundary tensor. In: Proceedings of Ninth IEEE International Conference on Computer Vision, ICCV (2003)

24. Knutsson, H., Andersson, M.: Implications of invariance and uncertainty for local structure analysis filter sets. Signal Processing: Image Communications 20(6), 569$581(2005)$

25. Nordberg, K.: A fourth order tensor for representation of orientation and position of oriented segments. Other academic, Linköping University, Department of Electrical Engineering, Sweden, diva2:288343 (2004)

26. Nordberg, K., Farnebäck, G.: Estimation of orientation tensors for simple signals by means of second-order filters. Signal Processing: Image Communication 20(6), 582-594 (2005)

27. Köthe, U., Felsberg, M.: Riesz-transforms versus derivatives: On the relationship between the boundary tensor and the energy tensor. In: Kimmel, R., Sochen, N.A., Weickert, J. (eds.) Scale-Space 2005. LNCS, vol. 3459, pp. 179-191. Springer, Heidelberg (2005)

28. Felsberg, M., Jonsson, E.: Energy tensors: Quadratic, phase invariant image operators. In: Kropatsch, W.G., Sablatnig, R., Hanbury, A. (eds.) DAGM 2005. LNCS, vol. 3663, pp. 493-500. Springer, Heidelberg (2005)

29. Felsberg, M., Köthe, U.: GET: The Connection Between Monogenic Scale-Space and Gaussian Derivatives. In: Kimmel, R., Sochen, N.A., Weickert, J. (eds.) ScaleSpace 2005. LNCS, vol. 3459, pp. 192-203. Springer, Heidelberg (2005)

30. Herberthson, M., Brun, A., Knutsson, H.: Representing pairs of orientations in the plane. In: Ersbøll, B.K., Pedersen, K.S. (eds.) SCIA 2007. LNCS, vol. 4522, pp. 661-670. Springer, Heidelberg (2007)

31. Barmpoutis, A., Vemuri, B.C., Forder, J.R.: Registration of high angular resolution diffusion MRI images using 4 order tensors. In: Ayache, N., Ourselin, S., Maeder, A. (eds.) MICCAI 2007, Part I. LNCS, vol. 4791, pp. 908-915. Springer, Heidelberg (2007)

32. Wang, Q., Ronneberger, O., Burkhardt, H.: Fourier analysis in polar and spherical coordinates. Technical Report Internal Report 1/08, IIF-LMB, Computer Science Department, University of Freiburg (2008)

33. Westin, C.-F., Knutsson, H.: Representation and Estimation of Tensors-Pairs. In: Visualization and Processing of Tensor Fields: Proceedings of the Dagstuhl Workshop (2010) submitted 